\title{
ON INTEGRAL EQUATIONS WITH DISCONTINUOUS KERNELS*
}

\author{
BY \\ J. D. TAMARKIN AND RUDOLPH E. LANGER
}

1. Introduction. In recent publications the authors have individually considered boundary value and expansion problems arising from the integral equation of the type

$$
y(t)=\rho \int_{\alpha}^{\beta} \Gamma(t, \tau) y(\tau) d \tau,
$$

when the kernel presents certain features of finite discontinuity along the line $t=\tau$. Kernels of this kind are typified by the Green's functions of ordinary differential equations with boundary conditions, and it was first noticed by Birkhoff that this fact might serve as a basis for the development of a theory of integral equations of this type. The discussion of the case in which the kernel itself is discontinuous was given by Langer $\dagger$ and more recently the discussion of the case in which the discontinuities occur in the first partial derivatives of the kernel, the kernel itself being continuous, was given by Langer jointly with Mrs. E. P. Brown. $\ddagger$ A history of the problem may be found in the introductions to these papers. The method of attack in each case depends upon a transformation of the integral equation into an integro-differential system.

A theory of integro-differential systems composed of an equation with boundary conditions has meanwhile been given independently by Tamarkin, $\S$ who showed also the applicability of this theory to the integral equation in which the kernel is discontinuous by giving a reduction (distinct from that of the papers mentioned above) of the integral equation to a system of the type considered.

The present paper is given to the discussion of the equation of type (1) in which the kernel and its partial derivatives of order $(n-2)$ are continuous

\footnotetext{
* Presented to the Society, December 28, 1926; received by the editors in December, 1927.

$\dagger$ On the theory of integral equations with discontinuous kernels, these Transactions, vol. 28 (1926): pp. 585-639.

¥On a class of integral equations with discontinuous kernels, these Transactions, vol. 29 (1927), pp. 683-715.

8 The notion of the Green's function in the theory of integro-differential equations, these Transactions, vol. 29 (1927), pp. 755-800.
} 
and the discontinuities along the line $t=\tau$ occur in the partial derivatives of higher order. Of the papers mentioned above those by Tamarkin and Langer respectively will be referred to in the text by $[T]$ and by $[L]$. By $[B]$ we refer to a paper by Birkhoff* of which we make frequent use.

2. The given equation. We consider the integral equation (1) supposing the kernel to be possessed of the following properties in the region

$$
\alpha \leqq{ }_{\tau}^{t} \leqq \beta .
$$

(i) The kernel and its partial derivatives to those of order $(n-2)$ $(n \geqq 2)$ are continuous in $(t, \tau)$.

(ii) The partial derivatives of orders $(n-1)$ to $3 n$ exist, are continuous except on the line $t=\tau$, and as the point $(t, \tau)$ approaches any point $\left(t_{0}, t_{0}\right)$ along a path having no points in common with the line $t=\tau$ these derivatives tend to definite limiting values which are independent of the path and are continuous in $t$.

$$
\left.\frac{\partial^{n-1} \Gamma(t, \tau)}{\partial t^{n-1}}\right]_{\tau=t}^{r-t+} \equiv \phi(t) \neq 0 .
$$

Under these hypotheses the function $\phi(t)$ is continuous and we may assume without loss of generality that

$$
\phi(t) \geqq \phi>0,
$$

since this involves at most a change in the sign of $\rho$.

3. The normalization of the equation. Since $\phi(t)$ satisfies the relation (2) its integral is monotonic increasing, and hence, the independent variable of the given equation may be changed by the substitution [ $\mathrm{L}, \S 3]$

$$
x=\frac{1}{l} \int_{\alpha}^{l} \phi(t)^{1 / n} d t, \quad \xi=\frac{1}{l} \int_{\alpha}^{\tau} \phi(\tau)^{1 / n} d \tau,
$$

where

$$
l=\int_{\alpha}^{\beta} \phi(t)^{1 / n} d t .
$$

This reduces the equation to the form

$$
u(x)=\lambda \int_{0}^{1} K(x, \xi) u(\xi) d \xi,
$$

* Boundary value and expansion problems etc., these Transactions, vol. 9 (1908), pp. 373-395. 
where we have set

$$
\begin{aligned}
K(x, \xi) & \equiv l^{-n+1} \Gamma(t, \tau) \phi(\tau)^{-1 / n}, \\
\lambda & =l^{n} \rho, \\
u(x) & \equiv y(t), \quad u(\xi) \equiv y(\tau) .
\end{aligned}
$$

We shall designate the equation in the form (4) as the "normalized equation." The properties of its kernel are readily found to be the following:

(A) The kernel and its partial derivatives to those of order $(n-2)$ are continuous in $(x, \xi)$.

(B) The partial derivatives of orders $(n-1)$ to $(2 n+1)$ exist and are continuous except for $x=\xi$, and as the point $(x, \xi)$ approaches any point $\left(x_{0}, x_{0}\right)$ along a path having no points in common with the line $x=\xi$ these derivatives tend to definite limiting values which are independent of the path and are continuous in $x$.

(C) If we abbreviate by setting

$$
\left.J_{i, j} f \equiv D_{x}^{i} D_{\xi}^{i} f(x, \xi)\right]_{\xi-\infty-\infty}^{\xi-x+}
$$

then

$$
J_{n-1-,, 8} K \equiv(-1) \cdot \quad(s=0,1,2, \cdots, n-1) .
$$

The proof of (C) is easily made to depend on the following lemma [L, 2].*

LEMma. If $F(t, \tau)$ is any function whose partial derivatives of the first order are possessed of property (ii) above, then

$$
\frac{d F(t, t \pm)}{d t}=F_{t}(t, t \pm)+F_{r}(t, t \pm)
$$

4. Transformation of the integral equation into an integro-differential equation. $\dagger$ In virtue of the properties of the kernel $K(x, \xi)$ we find by differentiation of equation (4) that

$$
\begin{aligned}
& u^{(s)}(x)=\int_{0}^{1} D_{x}^{*} K(x, \xi) \lambda u(\xi) d \xi, \quad \text { for } s=0,1,2, \cdots, n-1, \\
& u^{(n)}(x)=-\lambda u(x)+\int_{0}^{1} D_{x}^{n} K(x, \xi) \lambda u(\xi) d \xi .
\end{aligned}
$$

* Cf. also Hammerstein, Uber die asymptotische Darstellung der Eigenfunktionen linearer Integralgleichungen, Mathematische Annalen, vol. 93 (1924), p. 113.

$\dagger$ The method is that of $[T, 8 \$ 29-31]$. Cf. also the method of achieving such a transformation in [L, Ch. 3] and Langer and Brown, loc. cit., Ch. 3. 
We shall make now the further hypothesis

(iv) The given equation is such that the kernel

$$
E(x, \xi) \equiv D_{x}^{n} K(x, \xi)
$$

is possessed of a reciprocal kernel $\xi(x, \xi)$, defined by

$$
E(x, \xi)+\mathbb{E}(x, \xi)=\int_{0}^{1} E(x, s) \mathbb{E}(s, \xi) d s=\int_{0}^{1} \mathfrak{E}(x, s) E(s, \xi) d s .
$$

Then considering (6) formally as an equation in $\lambda u(x)$ with $u^{(n)}(x)$ known, we may write it in the "solved" form

$$
\lambda u(x)=-u^{(n)}(x)+\int_{0}^{1}\left(\xi(x, \xi) u^{(n)}(\xi) d \xi .\right.
$$

It is readily verified that $\mathscr{E}(x, \xi)$ possesses the property $(\mathrm{B})$ of the normalized kernel with $(2 n+1)$ replaced by $(n+1)$. The repeated integration by parts of the integral in (7) yields for that equation the form

(8) $L(u)+\lambda u=\sum_{i=1}^{n}\left\{u^{(i-1)}(0) \phi_{0, i}(x)+u^{(i-1)}(1) \phi_{1, i}(x)\right\}+\int_{0}^{1} f(x, \xi) u(\xi) d \xi$,

where

$$
\begin{aligned}
L(u) & =u^{(n)}(x)+p_{1}(x) u^{(n-1)}(x)+\cdots+p_{n}(x) u(x) \\
p_{i}(x) & =(-1)^{i-1} J_{0, i-1}(\xi \\
\phi_{0, i}(x) & =-(-1)^{n-i} D_{\xi}^{n-i} \mathbb{E}(x, 0), \phi_{1, i}(x)=(-1)^{n-i} D_{\xi}^{n-i} \mathbb{E}(x, 1), \\
f(x, \xi) & =(-1)^{n} D_{\xi^{n}}^{n} \mathbb{E}(x, \xi) .
\end{aligned}
$$

5. The boundary conditions. Every solution of (4) thus satisfies also the equation (8). In addition, however, it satisfies certain boundary conditions which may be obtained as follows.* Set $x=0$ in equations (5) and substitute in the integral the value of $\lambda u(\xi)$ as given formally by solving (8) for the second term on its left. With the use of Green's identity

$$
\int_{0}^{1}\left\{v L(u)-u L^{\prime}(v)\right\} d x=P(u, v)
$$

where $L^{\prime}$ is the operator adjoint to $L$, and $P(u, v)$ is the corresponding bilinear form in $u^{(j)}(0), u^{(j)}(1)$ and $v^{(j)}(0), v^{(j)}(1), j=1,2, \cdots,(n-1)$, we readily obtain the result in the form

* L, $\$ \S 10,31 ; \mathrm{T}, \S 29$. 


$$
L_{i}(u)=\int_{0}^{1} \alpha_{i}(\xi) u(\xi) d \xi \quad(i=1,2, \cdots, n),
$$

where

$$
\begin{aligned}
L_{i}(u)= & u^{(i-1)}(0)+P\left(u, K_{i}\right)-\sum_{j=1}^{n}\left\{u^{(j-1)}(0) \int_{0}^{1} K_{i}(\xi) \phi_{0, j}(\xi) d \xi\right. \\
& \left.+u^{(j-1)}(1) \int_{0}^{1} K_{i}(\xi) \phi_{1, j}(\xi) d \xi\right\} \\
\alpha_{i}(\xi)= & -L^{\prime}\left(K_{i}\right)+\int_{0}^{1} K_{i}(t) f(t, \xi) d t \\
K_{i}(\xi) \equiv & D_{x}^{i-1} K(0, \xi) .
\end{aligned}
$$

We state this result as follows.

THEOREM 1. Every solution of the normalized integral equation (4) is also a solution of the integro-differential system composed of the equation (8) and the boundary conditions (9).

6. The equivalence of the integro-differential system and the integral equation. We consider now the non-homogeneous system

$$
\begin{aligned}
L(u)+\lambda u=f(x) & +\sum_{j=1}^{n}\left\{u^{(j-1)}(0) \phi_{0, j}(x)+u^{(j-1)}(1) \phi_{1, j}(x)\right\} \\
& +\int_{0}^{1} f(x, \xi) u(\xi) d \xi
\end{aligned}
$$

together with the boundary conditions (9). The equation (11) differs from (8) only in the presence of the term $f(x)$ which is taken to be an arbitrary continuous function. We shall show that this system is equivalent to a non-homogeneous integral equation of the second kind.

Since (8) and (7) are equivalent forms of the same equality it is clear that (11) is equivalent to the equation

$$
u^{(n)}(x)=f(x)-\lambda u(x)+\int_{0}^{1}\left(\xi(x, \xi) u^{(n)}(\xi) d \xi,\right.
$$

and since $E(x, \xi)$ is the resolvent kernel of $E(x, \xi)$ this equation may be "solved" to give

$$
u^{(n)}(x)=f(x)-\lambda u(x)-\int_{0}^{1} E(x, \xi)\{f(\xi)-\lambda u(\xi)\} d \xi .
$$


Recalling that (6) is obtained from (4) by an $n$-fold differentiation it becomes clear that (12) may be written

$$
u^{(n)}(x)=\frac{d^{n}}{d x^{n}} \int_{0}^{1} K(x, \xi)\{\lambda u(\xi)-f(\xi)\} d \xi .
$$

Let the values (10) be substituted now into the equations (9). These become then

$$
\begin{aligned}
u^{(i)}(0)=\int_{0}^{1} D_{i}^{i} K(0, \xi)\{-L(u) & +\sum_{j=1}^{n}\left[u^{(j-1)}(0) \phi_{0, j}(\xi)+u^{(j-1)}(1) \phi_{1, j}(\xi)\right] \\
& \left.+\int_{0}^{1} f(\xi, t) u(t) d t\right\} d \xi,
\end{aligned}
$$

or, in virtue of (11),

$$
u^{(i)}(0)=\int_{0}^{1} D_{x}^{i} K(0, \xi)\{\lambda u(\xi)-f(\xi)\} d \xi \quad(i=0,1,2, \cdots, n-1) .
$$

With this fact it becomes clear that in each of the first $n$ successive integrations of (13) the constant of integration is zero, so that we obtain from (13)

$$
u(x)=F(x)+\lambda \int_{0}^{1} K(x, \xi) u(\xi) d \xi,
$$

where

$$
F(x)=-\int_{0}^{1} K(x, \xi) f(\xi) d \xi
$$

Hence every solution of the system (11), (9) is also a solution of equation (14).

Lastly it is clear that the system composed of (11) and (9) is not intrinsically altered if we replace in (11) every quantity of the set $u^{(j)}(0), u^{(j)}(1)$, $j=0,1,2, \cdots,(n-1)$, which is expressible linearly in terms of the quantities $L_{i}(u)$, by their values as given by (9). If this is done, the system (11), (9) may be written in the form

(a) $\quad L(u)+\lambda u=f(x)+\sum_{j=0}^{m} l_{j}(u) \Phi_{j}(x)+\int_{0}^{1} h(x, \xi) u(\xi) d \xi$,

$$
L_{i}(u)=\int_{0}^{1} \alpha_{i}(\xi) u(\xi) d \xi \quad(i=1,2, \cdots, n),
$$

where the $l_{j}(u), j=1,2, \cdots, m$, are simply those of quantities $u^{(k)}(0)$, $u^{(k)}(1)$ which are linearly independent of the quantities $L_{i}(u)$.

We have shown, then, that every solution of (15) is a solution of (14). The converse is easily shown by treating equation (14) as the corresponding 
homogeneous equation (4) was treated in $\$ 4$. Hence we may state the following theorem:

THEOREM 2. Under the hypothesis (iv) the integral equation (14) and the integro-differential system (15) are equivalent in the sense that every solution of either is also a solution of the other.

CoROLLARY. Under the hypothesis (iv) the given integral equation (4) is equivalent to the homogeneous differential system obtained from (15) by setting $f(x) \equiv 0$.

7. The Green's function of system (15). The integro-differential system of a type under which system (15) is included has been studied in paper [T]. To ascertain the availability of the results obtained there we must consider the hypotheses made [T: $\S 10, \mathrm{~A}, \mathrm{i}-\mathrm{v} ; \S 13, \mathrm{~B}, \mathrm{i}-\mathrm{ii} ; \S 19, \mathrm{C}, \mathrm{i}-\mathrm{v}$ ] and whether they are satisfied by the system in hand. Due to the specialized structure of system (15) it is readily found that of these hypotheses some [T: A, i, iii ; B, i; C, i-iii, v] are automatically satisfied. To meet the conditions [T: A, ii, v; C, iv] we must make the following hypothesis:

(v) The partial derivatives of order $(2 n+1)$ of $K(x, \xi)$ are of bounded variation in $\xi$ for fixed $x$ and in $x$ for fixed $\xi$, and are of uniformly bounded total variation on $(0,1)$.

To meet the remaining conditions [T: A, iv; B, ii] we must suppose further that the given integral equation is such that

(vi) The auxiliary differential system

$$
\begin{aligned}
L(u)+\lambda u & =f(x), \\
L_{i}(u) & =0
\end{aligned}
$$$$
(i=1,2, \cdots, n)
$$

is regular in the sense of Birkhoff [B, pp. 382-383].

By Theorem 2 the system (15) and the equation (14) have the same characteristic values. Let us suppose that $\lambda$ is not such a value. Then the system (15) admits of a unique solution [ $T$, Theorems 1, 2, 3] which is expressible in the form

$$
u(x)=\int_{0}^{1} \Gamma(x, t, \lambda) f(t) d t,
$$

where $\Gamma(x, t, \lambda)$ is the Green's function of system (15).* On the other hand since $\lambda$ is not a characteristic value the equation (14) possesses a resolvent kernel $\Omega(x, t, \lambda)$ in terms of which the equation may be written

* The notation here differs slightly from that in [T, Ch. 1] where the Green's function is written $\Gamma(x, t, \rho)$ while $\rho^{n}=\lambda$. 


$$
u(x)=F(x)-\lambda \int_{0}^{1} \Omega(x, t, \lambda) F(t) d t .
$$

Substituting in this the value of $F(x)$, and recalling the relation

we find that

$$
K(x, t)+\Re(x, t, \lambda)=\lambda \int_{0}^{1} \Re(x, \xi, \lambda) K(\xi, t) d \xi,
$$

$$
u(x)=\int_{0}^{1} \Re(x, t, \lambda) f(t) d t .
$$

Now $\Gamma(x, t, \lambda)$ and $\Re(x, t, \lambda)$ are both meromorphic functions of $\lambda$ with poles at the characteristic values of system (15). From this fact together with the fact that the right hand members of (16) and (17) are identical for an arbitrary choice $f(t)$, it follows that

$$
\Gamma(x, t, \lambda) \equiv \Omega(x, t, \lambda) .
$$

8. The characteristic values. We consider now in conjunction with the system (15) the associated differential system (15'). If we denote the Green's function of $\left(15^{\prime}\right)$ by $\Gamma^{\prime}(x, t, \lambda)$ we find directly that $\left(15^{\prime}\right)$ is expressible in the form of the integral equation

$$
u(x)=F^{\prime}(x)+\lambda \int_{0}^{1} K^{\prime}(x, t) u(t) d t
$$

where

$$
K^{\prime}(x, t) \equiv-\Gamma^{\prime}(x, t, 0)
$$

and

$$
F^{\prime}(x) \equiv-\int_{0}^{1} K^{\prime}(x, t) f(t) d t
$$

There is no loss in generality in supposing that $\lambda=0$ is not a characteristic value of system $\left(15^{\prime}\right)$, since a translation of the plane would not affect the character of the system.

It is clear that the resolvent kernel of $K^{\prime}(x, t)$ is $\Gamma^{\prime}(x, t, \lambda)$. Hence if $\lambda=\lambda_{\text {! }}^{\prime}$ is any characteristic value of the equation (14') we draw from the theory of the Fredholm equation* the fact that the coefficient of $\left(\lambda-\lambda_{\nu}^{\prime}\right)^{-1}$ in the expansion of $\Gamma^{\prime}(x, t, \lambda)$ in ascending powers of $\left(\lambda-\lambda_{\nu}^{\prime}\right)$ is

$$
\sum_{i=1}^{\infty} \phi_{i}^{\prime}(x) \psi_{i}(t)
$$

where $\left\{\phi_{i}, \psi_{i}\right\}$ is the biorthogonal set of fundamental functions of (14')

* Cf. Lalesco, Introduction d la Théorie des Equations Intégrales, Paris, 1912, pp. 40-59. 
corresponding to $\lambda=\lambda_{!}^{\prime} . *$ These functions are characteristic functions, i.e. solutions of the equation, when and only when $\lambda=\lambda_{\text {! }}^{\prime}$ is a simple pole of $\Gamma^{\prime}(x, t, \lambda)$. The integer $\sigma_{n}^{\prime}$ is called the multiplicity of the corresponding characteristic value.

Let the subscripts be assigned to the characteristic values now so that

$$
\left|\lambda_{\mathbf{i}}^{\prime}\right| \leqq\left|\lambda_{\mathbf{2}}^{\prime}\right| \leqq \cdots,
$$

each value $\lambda_{\nu}^{\prime}$ being repeated in this array a number of times equal to its index of multiplicity. The corresponding biorthogonal system of fundamental functions we designate by

$$
\left\{u_{\nu}^{\prime}(x), v_{\nu}^{\prime}(x)\right\} \quad(\nu=1,2, \cdots) .
$$

In similar fashion we arrange the characteristic values and fundamental functions of equation (14) in the array

$$
\begin{gathered}
\left|\lambda_{1}\right| \leqq\left|\lambda_{2}\right| \leqq \cdots, \\
\left\{u_{v}(x), v_{v}(x)\right\} .
\end{gathered}
$$

Let the positive constart $\delta$ be chosen now arbitrarily small and let the circles $c_{v}^{\prime}$ with radii $\delta$ and centers at the points $\lambda_{v}^{\prime}$ be drawn in the $\lambda$ plane. It is known [T, §23] that there exist infinitely many characteristic values $\lambda_{\nu}$ and moreover that all those characteristic values which exceed in absolute value a certain constant $\Lambda$ (which depends on $\delta$ ) lie within the circles $c_{v}^{\prime}$.

Due to the similarities of structure of the systems (15) and $\left(15^{\prime}\right)$ the statement above may be made somewhat more precise. The set of values $\left(17^{\prime}\right)$ are given by formulas [B, p. 383-385]

$$
\lambda_{v}^{\prime}=-( \pm 2 \nu \pi i)^{n}\left(1+\frac{\alpha}{\nu}+\frac{E}{\nu^{2}}\right),
$$

where $\alpha$ is a constant. Hence the constant $\delta$ may be chosen so small that for $\left|\lambda_{!}^{\prime}\right|$ sufficiently large no two of the small circles $c_{v}^{\prime}$ have points in common. Then since the residue of $\Gamma^{\prime}(x, t, \lambda)$ at $\lambda_{v}^{\prime}$ is given by (18), the set $\left\{\phi_{i}, \psi_{i}\right\}$ being biorthogonal we have

$$
\frac{1}{2 \pi i} \int_{c_{\nu}^{\prime}} \int_{0}^{1} \Gamma^{\prime}(x, x, \lambda) d x d \lambda=\sigma_{\nu}^{\prime} .
$$

Similarly we have

$$
\frac{1}{2 \pi i} \int_{c_{\nu}^{\prime}} \int_{0}^{1} \Gamma(x, x, \lambda) d x d \lambda=\sigma_{\nu},
$$

* These functions are called principal functions in the discussion of a more general boundary problem not linear in $\lambda$. Cf. Tamarkin, Some general problems of the theory of ordinary linear differential equations, etc., Mathematische Zeitschrift, vol. 27 (1927), pp. 1-54. 
and $\sigma_{v}$ represents the number of members of the set (19) which lie within the circle $c_{v}^{\prime} ; \sigma_{v}^{\prime}$ represents, of course, the number of members in set (17') which lie in $c_{r}^{\prime}$.

On the other hand if we set $\rho^{n}=\lambda$ and consider for the moment the situation in the $\rho$-plane, it is known [T, $\$ \S 19-21]$ that the dominant terms of $\Gamma^{\prime}(x, t, \lambda)$ and $\Gamma(x, t, \lambda)$ are the same (they depend only on the differential operator $L(u)$ ) provided that $|\rho|$ is sufficiently large and that $|\rho-\bar{\rho}|$ is uniformly bounded from zero, where $\bar{\rho}$ is any characteristic value $\rho_{v}^{\prime}$ or $\rho_{v}$. Moreover, in this case the difference of these Green's functions is of the order of $\rho^{-n}$. Hence we may write, when $\lambda$ is a point of a circle $c_{\nu}^{\prime}$, and $|\lambda|$ is sufficiently large,

$$
\left|\Gamma^{\prime}(x, t, \lambda)-\Gamma(x, t, \lambda)\right|<N /|\lambda|,
$$

where $N$ is a constant. But from this and (21), (21') it follows that

$$
\left|\sigma_{v}^{\prime}-\sigma_{v}\right| \leqq N / r_{v}
$$

where $r_{\nu}$ is the minimum of $|\lambda|$ on the circle $c_{\nu}^{\prime}$. Since the numbers $\sigma_{\nu}^{\prime}$ and $\sigma_{\nu}$ are integers whereas $r_{\nu}$ increases indefinitely with $\nu$, it follows that for $\nu$ sufficiently large $\sigma_{r}^{\prime}=\sigma_{\nu}$, i.e. the number of values $\lambda_{i}$ counted with their multiplicities which lie in $c_{v}^{\prime}$ is equal to the multiplicity of $\lambda_{v}^{\prime}$ when $\left|\lambda_{r}^{\prime}\right|>\Lambda$.

As to the actual multiplicity of the value $\lambda_{v}^{\prime}$ we note that because of the hypothesis (vi) these values are in general simple and can be at most double. A necessary though not sufficient condition for double characteristic values is that $n$ be even and that the values $\theta_{i}$ defined by Birkhoff $[\mathrm{B}, \mathrm{p} .383]^{*}$ satisfy the relation

$$
\theta_{1}^{2}-4 \theta_{0} \theta_{2}=0 .
$$

We formulate these results as follows.

THEOREM 3. If the kernel of the normalized integral equation (4) satisfies the hypotheses (iv)-(vi), then there exist infinitely many characteristic values $\lambda_{j}, j=1,2, \cdots$. These values are represented asymptotically by the quantities $\lambda_{i}^{\prime}$ given by (20) in the sense that for $|\lambda|$ sufficiently large all values $\lambda_{j}$ lie within circles $c_{v}^{\prime}$ of arbitrarily small radii $\delta$ about the quantities $\lambda_{v}^{\prime}$ as centers. The characteristic values are in general simple and in general but one lies in a circle $c_{\nu}^{\prime}$. A necessary condition for double characteristic values or that circles $c_{\nu}^{\prime}$ enclose two characteristic values is that $n$ be even and that

$$
\theta_{1}^{2}-4 \theta_{0} \theta_{2}=0 \text {. }
$$

\footnotetext{
* Cf. also Tamarkin, Sur quelques points de la theorie des equations differentielles etc., Rendiconti del Circolo Matematico di Palermo, vol. 23 (1912), pp. 345-382.
} 
9. The characteristic functions. In the deduction of the asymptotic expressions for the characteristic functions the formal work for the case when the integer $n$ is odd differs to some extent from that for the case when $n$ is even. To avoid repetition we shall treat only the case in which $n$ is odd. The reader will find no difficulty with the parallel deductions for the omitted case.

By the corollary to Theorem 2 the given integral equation is equivalent to the homogeneous system (15). Let

$$
y_{1}(x), y_{2}(x), \cdots, y_{n}(x)
$$

be any fundamental set of solutions of the differential equation

$$
L(y)+\lambda y=0 .
$$

Further let $\delta(t)$ designate the determinant

$$
\delta(t) \equiv\left|\begin{array}{ccc}
y_{1}^{(n-1)}(t) & \cdots & y_{n}^{(n-1)}(t) \\
\vdots & \vdots \\
y_{1}(t) & \cdots & y_{n}(t)
\end{array}\right|,
$$

and $\delta_{j}(t)$ the cofactor in $\delta(t)$ of the element $y_{j}{ }^{(n-1)}(t)$. Then upon setting

$$
z_{k}(t)=\delta_{k}(t) / \delta(t) \quad(k=1,2, \cdots, n),
$$

the general solution of the non-homogeneous equation

$$
L(u)+\lambda u=\phi(x)
$$

may be written in the form

$$
u(x)=\sum_{j=1}^{n} c_{j} y_{j}(x)+\int_{0}^{1} \gamma(x, t) \phi(t) d t,
$$

where $c_{j}, j=1,2, \cdots, n$, are arbitrary constants, and

$$
\gamma(x, t)= \begin{cases}\sum_{j=1}^{\mu} y_{j}(x) z_{j}(t) & \text { for } t<x, \\ \sum_{j=\mu+1}^{n} y_{j}(x) z_{j}(t) & \text { for } t>x .\end{cases}
$$

In this $\mu$ may be chosen as any integer of the set $1,2, \cdots, n$.

Let $\lambda$ be chosen now as a characteristic value of equation (4) and set $\rho^{n}=\lambda$. Further let $\omega_{j}, j=1,2, \cdots, n$, designate the $n$ roots of the equation $\omega^{n}+1=0$, the subscripts being so assigned that

$$
R\left(\rho \omega_{1}\right) \leqq R\left(\rho \omega_{2}\right) \leqq \cdots \leqq R\left(\rho \omega_{n}\right) .
$$


The integer $\mu$ in (25) we shall choose so that

$$
R\left(\rho \omega_{\mu-1}\right)<0<R\left(\rho \omega_{\mu+1}\right) .
$$

There exists then [B, p. 391] a fundamental set of solutions $y_{j}(x)$ of equation (22) whose asymptotic form is given by the relations

$$
\begin{aligned}
& \frac{d^{s} y_{j}(x)}{d x^{s}}=e^{\rho \omega_{j} x} \rho^{s}\left[\omega_{j^{s}}^{s}\right], * \\
& \frac{d^{s} z_{j}(t)}{d t^{s}}=\frac{1}{n} e^{-\rho \omega_{j} t \rho^{s-n+1}}\left[\left(-\omega_{j}\right)^{s+1}\right] \\
& (s=0,1, \cdots, n-1) .
\end{aligned}
$$

We define the quantities $\tau_{s}(x, t)$ now by the formulas

$$
\tau_{s}(x, t)=\left\{\begin{array}{cl}
\sum_{j=1}^{\mu} e^{\rho \omega_{j}(x-t)}\left[\frac{-\omega_{j}^{8}}{n}\right], & \text { when } t<x, \\
\sum_{j=\mu+1}^{n} e^{\rho \omega_{j}(x-t)}\left[\frac{\omega_{j}^{8}}{n}\right], & \text { when } t>x .
\end{array}\right.
$$

Then we obtain from (25) and (26) the relation

$$
\gamma(x, t)=\rho^{-n+1} \tau_{1}(x, t) .
$$

Now by Theorem $3,\left|\rho-\rho_{\prime}^{\prime}\right|$ is uniformly small when $|\rho|$ is sufficiently large, $\rho_{\nu}^{\prime n}=\lambda_{\nu}^{\prime}$ being a characteristic value of the system $\left(15^{\prime}\right)$. With the use of formula [B, p. 385]

$$
\rho_{\nu}^{\prime}=\frac{2 \nu \pi i}{\omega_{\mu}}\left\{1+\frac{a}{\nu}+\frac{E}{\nu^{2}}\right\},
$$

$a$ being a constant, it is readily found therefore that the quantities

$$
\begin{aligned}
e^{\rho \omega_{j}(x-t)}, & t<x \text { for } j=1,2, \cdots, \mu, \\
& t>x \text { for } j=\mu+1, \cdots, n,
\end{aligned}
$$

are uniformly bounded.

Returning to equation (23) let $\phi(x)$ be chosen as the function

$$
\phi(x)=\sum_{j=1}^{m} c_{j}^{\prime} \Phi_{j}(x)+\int_{0}^{1} h(x, \xi) u(\xi) d \xi,
$$

(b)

$$
c_{j}^{\prime}=l_{j}(u),
$$

$$
(j=1,2, \cdots, m)
$$

- We use here the notation of $[B$, p. 389] wherein $[\omega]$ designates an expression of the form $\omega+E^{\prime} \rho$ and $E$ is bounded for $|\rho|$ large. 
the quantities involved being those which occur also in equation (15a). The equation (23) becomes then identically the equation (15a) with $f(x) \equiv 0$. Hence it is found on substituting the forms (25) in equation (24) that the solution of the homogeneous equation (15a) satisfies the relation

$$
u(x)=\sum_{j=1}^{n} c_{j} y_{j}(x)+\sum_{j=1}^{m} c_{j}^{\prime} \Psi_{j}(x)+\int_{0}^{1} H(x, \xi) u(\xi) d \xi,
$$

where

$$
\begin{aligned}
\Psi_{j}(x) & =\int_{0}^{1} \gamma(x, t) \Phi_{j}(t) d t \\
H(x, \xi) & =\int_{0}^{1} \gamma(x, t) n(t, \xi) d t .
\end{aligned}
$$

Under the hypothesis (v) the functions $\Phi_{j}(t)$ are continuous and have derivatives of bounded variation on $(0,1)$, while $h(t, \xi)$ as a function of $t$ with $\xi$ fixed has the same properties on each of the sub-intervals $(0, \xi)$ and $(\xi, 1)$.

Consider the integral $H(x, \xi)$. Integrating the formula (32) by parts we obtain

$$
\begin{aligned}
H(x, \xi)= & -\rho^{-n}\left\{-h(x, \xi)[1]+\tau_{0}(x, \xi)[h(\xi-, \xi)-h(\xi+, \xi)]\right. \\
& \left.-\tau_{0}(x, 0) h(0, \xi)+\tau_{0}(x, 1) h(1, \xi)-\int_{0}^{1} \tau_{0}(x, t) h_{t}(t, \xi) d t\right\}
\end{aligned}
$$

and differentiation of this leads to the formulas

$$
\begin{aligned}
\frac{\partial \cdot H(x, \xi)}{\partial x^{\imath}}= & -\rho^{-n+\cdot}\left\{\tau_{s}(x, \xi)[h(\xi-, \xi)-h(\xi+, \xi)]-\tau_{s}(x, 0) h(0, \xi)\right. \\
& \left.+\tau_{s}(x, 1) h(1, \xi)-\int_{0}^{1} \tau_{s}(x, t) h_{t}(t, \xi) d t\right\} \\
& \quad(s=1,2, \cdots, n-1) .
\end{aligned}
$$

Since $h_{t}(t, \xi)$ is of bounded variation in $t$ we may apply the second law of the mean to show that the integrals remaining in these formulas are of the form $E / \rho$. Hence we find that

$$
\frac{\partial^{s} H(x, \xi)}{\partial x^{s}}=\frac{E}{\rho^{n-s}} \quad(s=0,1,2, \cdots, n-1) .
$$

The formula for $\Psi_{j}(x)$ in (32) may be treated precisely as we have treated that for $H(x, \xi)$. Recalling that $\Phi_{j}(t)$ is continuous throughout $(0,1)$ we find from the formulas analogous to (33) and (34) that 


$$
\begin{aligned}
\frac{d^{\imath} \Psi_{j}(x)}{d x^{*}}=\rho^{-n+\varepsilon}\left\{\delta_{0_{e}} \Phi_{j}(x)+\tau_{s}(x, 0) \Phi_{j}(0)-\tau_{s}(x, 1) \Phi_{j}(1)+\frac{E}{\rho}\right\}^{*} & \\
& (s=0,1,2, \cdots, n-1) .
\end{aligned}
$$

From (35) it is seen directly that the kernel $H(x, \xi)$ of the integral equation (31) possesses a reciprocal kernel $\mathfrak{W}(x, \xi)$ for all values of $\rho$ sufficiently large in absolute value. In "solved" form, then, equation (31) may be written

$$
u(x)=\sum_{j=1}^{n} c_{j} Y_{j}(x)+\sum_{j=1}^{m} c_{i}^{\prime} \Omega_{j}(x)
$$

where

$$
\begin{aligned}
& Y_{j}(x)=y_{j}(x)-\int_{0}^{1} \mathfrak{W}(x, \xi) y_{j}(\xi) d \xi \\
& \Omega_{j}(x)=\Psi_{j}(x)-\int_{0}^{1} \mathfrak{W}(x, \xi) \Psi_{j}(\xi) d \xi .
\end{aligned}
$$

From the Neumann series for $\mathfrak{Q}(x, \xi)$, i.e.

$$
\begin{aligned}
\mathscr{W}(x, \xi)=-H(x, \xi) & -\int_{0}^{1} H(x, t) H(t, \xi) d t \\
& -\int_{0}^{1} \int_{0}^{1} H\left(x, t_{1}\right) H\left(t_{1}, t_{2}\right) H\left(t_{2}, \xi\right) d t_{1} d t_{2}-\cdots,
\end{aligned}
$$

together with formula (35) it is evident that

$$
\frac{\partial^{\prime} \mathfrak{Q}(x, \xi)}{\partial x^{s}}=\frac{E}{\rho^{n-s}} \quad(s=0,1,2, \cdots, n-1) .
$$

In consequence of this and (36) we see that

$$
\begin{aligned}
& \frac{d^{\bullet} \Omega_{j}(x)}{d x^{\varepsilon}}=\rho^{-x+\imath}\left\{\delta_{0 \ell} \Phi_{j}(x)+\tau_{\imath}(x, 0) \Phi_{j}(0)-\tau_{\varepsilon}(x, 1) \Phi_{j}(1)+\frac{E}{\rho}\right\} \\
& \text { while from (26) } \\
& (s=0,1,2, \cdots \cdot n-1) \text {, }
\end{aligned}
$$

$$
\frac{d^{\bullet} Y_{j}(x)}{d x^{*}}=\rho^{e} e^{\rho \omega_{j} x}\left[\omega_{j}^{*}\right]
$$

For the evaluation of the characteristic function $u(x)$ from formula (37) it is still necessary to determine the $n+m$ constants $c_{j}$ and $c_{j}^{\prime}$. For

$$
* \delta_{i j}=\left\{\begin{array}{lll}
0 & \text { if } i \neq j \\
1 & \text { if } & i=j
\end{array}\right.
$$


this dutermination we have the equations (15b) and (30b) which become on substituting in them the value of $u(x)$ as given by (37)

$$
\sum_{k=1}^{n} m_{i, k} c_{k}+\sum_{j=1}^{m} m_{i, j}^{\prime} c_{j}^{\prime}=0 \quad(i=1,2, \cdots, n+m),
$$

where

$$
\begin{aligned}
& m_{i, k}=\left\{\begin{array}{cl}
L_{i}\left(Y_{k}\right)-\int_{0}^{1} \alpha_{i}(\xi) Y_{k}(\xi) d \xi, & \text { when } i \leqq n, \\
-l_{p}\left(Y_{k}\right), & \text { when } i=n+p ;
\end{array}\right. \\
& m_{i, j}^{\prime}=\left\{\begin{array}{cl}
L_{i}\left(\Omega_{j}\right)-\int_{0}^{1} \alpha_{i}(\xi) \Omega_{j}(\xi) d \xi, & \text { when } i \leqq n, \\
\delta_{p i}-l_{p}\left(\Omega_{j}\right), & \text { when } i=n+p .
\end{array}\right.
\end{aligned}
$$

We may assume without loss of generality that the set of operators $L_{i}(u)$ have been reduced to the normal form [B, p. 382] in which

where

$$
L_{i}(u)=W_{i 0}(u)+W_{i 1}(u),
$$

$$
\begin{aligned}
& W_{i 0}(u)=a_{i} u^{\left(k_{i}\right)}(0)+\sum_{j=0}^{k_{i}-1} a_{i j} u^{(j)}(0), \\
& W_{i 1}(u)=b_{i} u^{\left(k_{i}\right)}(1)+\sum_{j=0}^{k_{i}-1} b_{i j} u^{(j)}(1),
\end{aligned}
$$

and

$$
n-1 \geqq k_{1} \geqq k_{2} \geqq \cdots \geqq k_{n} \text {. }
$$

On the other hand by the definition of the operators $l_{j}(u)$ we may assume the subscripts so assigned that

$$
l_{j}(u)=\left\{\begin{array}{ll}
\text { either } & u^{\left(h_{j}\right)}(0), \\
\text { or } \quad u^{\left(h_{j}\right)}(1),
\end{array} \quad \text { where } n-1 \geqq h_{1} \geqq h_{2} \geqq \cdots \geqq h_{m} .\right.
$$

Due to these peculiarities of structure of the operators involved we find readily from (43) with use of (40) that

$$
m_{i, j}^{\prime}=\left\{\begin{array}{l}
\rho^{k_{i}-n} E \text { for } i \leqq n \\
{\left[\delta_{p i}\right] \text { for } i=n+p}
\end{array}\right.
$$

Since $\alpha_{i}(\xi)$ possesses a derivative which is of bounded variation we find also from (41) that

$$
-\int_{0}^{1} \alpha_{i}(\xi) Y_{k}(\xi) d \xi= \begin{cases}E / \rho & \text { for } k=1,2, \cdots, \mu, \\ e^{\infty \infty_{k} E} / \rho \text { for } k=\mu+1, \cdots, n .\end{cases}
$$


Hence it follows that, for $i \leqq n$,

$$
m_{i, k}= \begin{cases}\left(\rho \omega_{k}\right)^{k_{i}}\left[a_{i}\right] & \text { when } k \leqq \mu-1, \\ \left(\rho \omega_{k}\right)^{k_{i}}\left\{\left[a_{i}\right]+e^{\rho \omega_{\mu}}\left[b_{i}\right]\right\} & \text { when } k=\mu, \\ \left(\rho \omega_{k}\right)^{k} e^{\rho \omega_{k}}\left[b_{i}\right] & \text { when } k \geqq \mu+1,\end{cases}
$$

while for $i=n+p$,

$$
m_{i, k}= \begin{cases}\left(\rho \omega_{k}\right)^{h_{p}}\left[a_{p}^{\prime}\right] & \text { when } k \leqq \mu-1, \\ \left(\rho \omega_{k}\right)^{h_{p}}\left\{\left[a_{p}^{\prime}\right]+e^{\rho \omega_{\mu}}\left[b_{p}^{\prime}\right]\right\} & \text { when } k=\mu, \\ \left(\rho \omega_{k}\right)^{h_{p}} e^{\rho \omega_{k}}\left[b_{p}^{\prime}\right] & \text { when } k \geqq \mu+1,\end{cases}
$$

every $a_{p}^{\prime}$ and $b_{p}^{\prime}$ being either 0 or 1 .

If we consider as unknowns in system (42) the quantities $c_{k}^{\prime \prime}$ in place of the quantities $c_{k}$ where

$$
c_{k}^{\prime \prime}=\left\{\begin{array}{l}
c_{k} \quad \text { when } k=1,2, \cdots, \mu, \\
c_{k} e^{\rho \omega_{k}} \text { when } k=\mu+1, \cdots, n,
\end{array}\right.
$$

and remove from the $i$ th equation, $i=1,2, \cdots, n$, the factor $\rho^{k_{i}}$, the matrix of the resulting system becomes

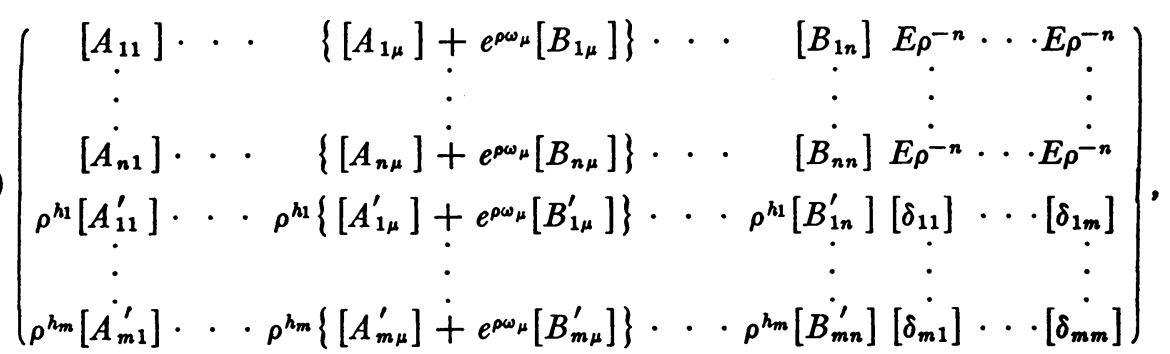

where the $A_{i j}, A_{i j}^{\prime}, B_{i j}, B_{i j}^{\prime}$ are constants. The equation $D(\rho)=0$, where $D(\rho)$ is the determinant of the matrix (44), is precisely the characteristic equation by which the characteristic numbers are determined. Since we suppose $\rho$ a characteristic number it follows that the system (42) admits of a solution in which at least one of the unknowns differs from zero.

The associated matrix for the differential system $\left(15^{\prime}\right)$ is readily shown to be

$$
\left\{\begin{array}{ccc}
{\left[A_{11}\right] \cdot \cdot} & \cdot\left\{\left[A_{1 \mu}\right]+e^{\rho \omega_{\mu}}\left[B_{1 \mu}\right]\right\} \cdot \cdot\left[B_{1 n}\right] \\
\vdots & \vdots \\
{\left[\dot{A}_{n 1}\right] \cdot \cdot} & \cdot\left\{\left[A_{n \mu}\right]+e^{\rho \omega_{\mu}}\left[B_{n \mu}\right]\right\} \cdot \cdot \cdot\left[\dot{B}_{n n}\right]
\end{array}\right\} \cdot
$$

Let the determinant of this matrix be $\Delta(\rho)$. Then $\Delta(\rho)=0$ is the characteristic equation of the differential system $\left(15^{\prime}\right)$. The condition (vi) that this dif- 
ferential system be regular implies that at least one of the cofactors of the elements of the $\mu$ th column of $\Delta(\rho)$ does not vanish. Due to the structural relationship of the matrices (44) and (45) it is readily seen to follow for $|\rho|$ sufficiently large at least one of the cofactors of the elements of the $\mu$ th column of $D(\rho)$ is different from zero. Hence the ratios of the unknowns $c_{k}^{\prime \prime}$ and $c_{j}^{\prime}, k=1,2, \cdots, n ; j=1,2, \cdots, m$, are obtainable from the ratios of the cofactors of a row of the determinant of (44). Substituting in these cofactors the value

given by (29), we may write

$$
e^{\rho \omega_{\mu}}=\left[e^{2 \pi i a / \omega_{\mu}}\right]
$$

$$
\begin{aligned}
c_{1}: c_{2}: \cdots: c_{\mu}: e^{\rho \omega_{\mu+1}} c_{\mu+1}: \cdots: e^{\rho \omega_{n}} c_{n}: c_{1}^{\prime}: \cdots: c_{m}^{\prime} \\
\quad=\left[Q_{1}\right]:\left[Q_{2}\right]: \cdots:\left[Q_{n}\right]: \rho^{n-1}\left[Q_{n+1}\right]: \cdots: \rho^{n-1}\left[Q_{n+m}\right],
\end{aligned}
$$

each $Q_{i}$ being a constant which may be computed from the elements of (44) and at least one $Q_{j}(j=1,2, \cdots, n)$ differing from zero. Upon substituting the quantities on the right of (46) for the coefficients in (37) and using (40) and (41) we find the form of the function $u(x)$. The result we state as follows.

THEOREM 4. If the normalized integral equation satisfies the hypotheses (iv)-(vi) the characteristic function $u(x)$ corresponding to the characteristic value $\lambda=\rho^{n}$ is of the form

$$
\begin{aligned}
u(x) & =\sum_{j=1}^{\mu} e^{\rho \omega_{j} x}\left[Q_{j}\right]+\sum_{j=\mu+1}^{n} e^{\rho \omega_{j}(x-1)}\left[Q_{j}\right], \\
\frac{d^{s} u(x)}{d x^{s}}=\rho^{s}\left\{\sum_{j=1}^{\mu} e^{\rho \omega_{j} x}\left[Q_{j} \omega_{j}^{s}\right]+\sum_{j=\mu+1}^{n} e^{\rho \omega_{j}(x-1)}\left[Q_{j} \omega_{j}^{s}\right]\right\} & (s=1,2, \cdots, n-1)
\end{aligned}
$$

the quantities $Q_{j}$ being constants.

10. The expansion of an arbitrary function. Let $f(x)$ be any function which is integrable on the interval $(0,1)$. We consider the following integrals

$$
\begin{aligned}
& I_{N}(f)=\frac{1}{2 \pi i} \int_{C_{N}} \int_{0}^{1} \Gamma(x, t, \lambda) f(t) d t d \lambda, \\
& I_{N}^{\prime}(f)=\frac{1}{2 \pi i} \int_{C_{N}} \int_{0}^{1} \Gamma^{\prime}(x, t, \lambda) f(t) d t d \lambda,
\end{aligned}
$$

where $\Gamma$ and $\Gamma^{\prime}$ are the Green's functions defined in $\$ \S 6$ and 7 , and $C_{N}$ is a circle $|\lambda|=R_{N}$ where $\left|\lambda_{N}\right|<R_{N}<\left|\lambda_{N+1}\right|{ }^{*} \quad$ The radius $R_{N}$ may be chosen

* If there are infinitely many double characteristic values, $N$ is to take on only those values for which this inequality is possible. 
moreover so that $C_{N}$ for every $N$ sufficiently large has no points in common with any of the small circles $c_{\nu}$, with radii $\delta$ and drawn with the points $\lambda_{\nu}$ as centers. It is known then [T, Theorem 6] that

$$
\lim _{N \rightarrow \infty}\left\{I_{N}(f)-I_{N}^{\prime}(f)\right\}=\left\{\begin{array}{l}
0 \quad \text { uniformly } 0<a \leqq x \leqq b<1, \\
\int_{0}^{1} T_{0}(t) f(t) d t \text { for } x=0, \\
\int_{0}^{1} T_{1}(t) f(t) d t \text { for } x=1,
\end{array}\right.
$$

where the functions $T_{0}(t)$ and $T_{1}(t)$ are known and do not depend on $f(x)$.

Now, if we remember the definition of the sets $\left\{u_{v}, v_{v}\right\},\left\{u_{r}^{\prime}, v_{v}^{\prime}\right\}$, we may write

$$
I_{N}(f)=\sum_{N}(f), \quad I_{M}^{\prime}(f)=\sum_{M}^{\prime}(f)
$$

where

$$
\begin{aligned}
& \sum_{N}(f)=\sum_{v=1}^{N} u_{i}(x) \int_{0}^{1} f(t) v_{\nu}(t) d t, \\
& \sum_{M}^{\prime}(f)=\sum_{v=1}^{M} u_{\nu}^{\prime}(x) \int_{0}^{1} f(t) v_{\nu}^{\prime}(t) d t,
\end{aligned}
$$

$M$ being the number of characteristic values $\lambda_{r}^{\prime}$ within the circle $C_{N}$. By Theorem 3 there exists a positive constant $R$ such that when $R_{N}>R$ the number of values $\lambda$, between the circle $C_{N}$ and the circle $C:|\lambda|=R$ is the same as the number of values $\lambda_{r}^{\prime}$ between these circles. From this it follows that $|N-M|$ is finite, not exceeding the number of characteristic values $\lambda$, within the circle $C$.

Consider now the identity

$$
\sum_{M}^{\prime}(f)-\sum_{N}^{\prime}(f)= \pm \sum_{,=N}^{M} \frac{1}{2 \pi i} \int_{c_{\nu^{\prime}}} \int_{0}^{1} \Gamma^{\prime}(x, t, \lambda) f(t) d t d \lambda .
$$

Since

$$
\left|\Gamma^{\prime}(x, t, \lambda)\right|<A|\lambda|-(n-1) / n,
$$

where $A$ is a constant [T, §18], it follows that each of the integrals on the right of (51) converges uniformly to zero as $N \rightarrow \infty$. We readily draw from this the result which we formulate as follows.

THEOREM 5. If the normalized integral equation satisfies the hypotheses (iv)-(vi) then the series

$$
\sum_{\infty}(f) \text { and } \sum_{\infty}^{\prime}(f)
$$


are equiconvergent for every integrable function $f(x)$ in the interior of the interval $(0,1)$, i.e.

$$
\lim _{N \rightarrow \infty}\left\{\sum_{N}(f)-\sum_{N}^{\prime}(f)\right\}=0 \text { uniformly for } 0<a \leqq x \leqq b<1 .
$$

At the end points $x=0$ and $x=1$ the difference of the series converges to

$$
\int_{0}^{1} T_{0}(t) f(t) d t \text { and } \int_{0}^{1} T_{1}(t) f(t) d t
$$

respectively, where $T_{0}(t)$ and $T_{1}(t)$ are known and do not depend on $f(x)$.*

The conclusions reached above enable us now to state also the following facts $[T, \S 35]$ :

THEOREM 6. If the normalized integral equation satisfies hypotheses (iv)(vi) with $n \geqq 2$, then when $\left|\lambda-\lambda_{\nu}\right| \geqq \delta$ and $|\lambda|$ is sufficiently large its kernel and its resolvent kernel admit of the absolutely and uniformly convergent expansions

$$
\Re(x, t, \lambda)=\sum_{v=1}^{\infty} \Omega_{v}(x, t, \lambda), \quad K(x, t)=-\sum_{v=1}^{\infty} \Omega_{v}(x, t, 0),
$$

where $\Omega_{\nu}(x, t, \lambda)$ is the principal part of $\Re(x, t, \lambda)$ at the pole $\lambda=\lambda_{\nu}$. The solution of the non-homogeneous equation is then given by

$$
u(x)=f(x)-\lambda \sum_{\nu=1}^{\infty} \int_{0}^{1} \Omega_{v}(x, t, \lambda) f(t) d t,
$$

the series converging uniformly and absolutely for every integrable function $f(x)$.

If all the characteristic values are simple, these expansions are, more explicitly,

$$
\begin{gathered}
\Re(x, t, \lambda)=\sum_{\nu=1}^{\infty} \frac{u_{\nu}(x) v_{\nu}(t)}{\lambda-\lambda_{\nu}}, \quad K(x, t)=\sum_{\nu=1}^{\infty} \frac{u_{\nu}(x) v_{\nu}(t)}{\lambda_{\nu}}, \\
u(x)=(f x)-\lambda \sum_{v=1}^{\infty} \frac{u_{\nu}(x)}{\lambda-\lambda_{\nu}} \int_{0}^{1} f(t) v_{\nu}(t) d t .
\end{gathered}
$$
\$3].

An analogous theorem has been stated also in the case where $n=1[\mathrm{~T}$,

* Let $\left\{u_{n}^{\prime \prime}, v_{v}^{\prime \prime}\right\}$ be the set of fundamental functions of the problem $L(u)+\lambda u=0$, $L_{i}(u)=\int_{0}^{1} \alpha_{i}(\xi) u(\xi) d \xi$. On setting $\sum_{N^{\prime \prime}}(f) \equiv \sum_{\nu-1}^{N} u_{\nu}^{\prime \prime}(x) \int_{0}^{1} f(t) v_{\nu}^{\prime \prime}(t) d t$, it is readily proved that the series $\sum_{N}(f)$ and $\sum_{N}(f)$ are equiconvergent on the whole of $(0,1)$, the end points inclusive.

BroWN UNIVERSITY,

Providence, R. I.;

UNIVERSITY OF WISCONSIN,

Madison, Wis. 